\title{
ARTICLE
}

Molecular Diagnostics

\section{The relationships between systemic cytokine profiles and inflammatory markers in colorectal cancer and the prognostic significance of these parameters}

\author{
Ji Won Park ${ }^{1,2}$, Hee Jin Chang ${ }^{2,3,4}$, Hyun Yang Yeo ${ }^{3}$, Nayoung Han ${ }^{4}$, Byung Chang Kim², Sun-Young Kong ${ }^{5}$, Jeongseon Kim (iD ${ }^{6}$ and \\ Jae Hwan $\mathrm{Oh}^{2}$
}

BACKGROUND: Immunomodulatory cytokines and systemic inflammatory markers are important during cancer development and progression. This study investigated the association and prognostic impact of systemic cytokine profiles and inflammatory markers in colorectal cancer (CRC).

METHODS: Interleukin (IL)-1 $\beta$, IL-6, IL-8, IL-9, IL-10, tumour necrosis factor (TNF)- $\alpha$ and vascular endothelial growth factor (VEGF) serum levels were measured using multiplex bead assays in CRC patients. Data on systemic inflammatory markers, such as the modified Glasgow prognostic score (mGPS), the neutrophil-to-lymphocyte ratio (NLR), platelet-to-lymphocyte ratio (PLR), lymphocyte-to-monocyte ratio (LMR), prognostic nutritional index (PNI) and fibrinogen, were collected. Survival analysis was performed to identify factors associated with progression-free survival (PFS) and overall survival (OS).

RESULTS: There were moderate-to-strong correlations within serum cytokines, as well as within systemic inflammatory markers, whereas the associations between serum cytokines and systemic inflammatory markers were generally weak. IL-8 and the LMR were independent significant prognostic factors for PFS and OS. The low IL-8 and high LMR group had the best survival (both PFS and OS) of all groups.

CONCLUSIONS: Systemic cytokine profiles and inflammatory markers have relatively weak intergroup correlations. A composite classification of systemic cytokine profiles and inflammatory markers has an enhanced prognostic value in CRC.

British Journal of Cancer (2020) 123:610-618; https://doi.org/10.1038/s41416-020-0924-5

\section{BACKGROUND}

Tumour pathologic characteristics are associated with prognosis in colorectal cancer (CRC). However, these characteristics alone do not accurately predict the survival outcomes of patients. Tumourassociated inflammation can also determine the prognosis of patients. This type of inflammation is known to occur as a local immune response and as systemic inflammation. ${ }^{1}$ As a part of the local immune response, the composition of tumour-infiltrating lymphocytes in the tumour microenvironment is correlated with prognosis in $\mathrm{CRC}^{2}$ As a part of systemic inflammation, circulating cytokines or systemic inflammatory markers have been suggested as prognostic markers in $\mathrm{CRC}^{3,4}$

Classic inflammatory cytokines, such as interleukin (IL)-1 $1 \beta$, IL-6 and tumour necrosis factor (TNF)- $\alpha$, activate the NF-KB and STAT3 signalling pathways, and induce the expression of genes that promote the invasion of cancer cells and angiogenesis. ${ }^{5}$ Several cytokines, especially IL-6, have been evaluated and found to be associated with survival in CRC. ${ }^{6}$ However, the association between these cytokines and outcomes was not consistent in previous reports. As an inflammatory chemokine, IL-8 induces the proliferation and migration of CRC cells by promoting neutrophil chemotaxis and angiogenesis. ${ }^{7}$ IL-8 has been suggested as a diagnostic marker and a prognostic factor for CRC. ${ }^{8}$ As a cytokine associated with a variety of inflammatory and autoimmune diseases, IL-9 may have a dual role in CRC progression. ${ }^{9}$ Vascular endothelial growth factor (VEGF) promotes tumour angiogenesis, and is the most potent angiogenic growth factor. High serum VEGF levels were associated with poor survival in CRC. ${ }^{10,11}$

Systemic inflammatory markers have been introduced as an integrative method to measure systemic immunity. ${ }^{12}$ These markers include inflammation-associated cell enumeration or serum inflammatory markers, such as C-reactive protein (CRP). ${ }^{13}$ Inflammatory markers, such as the neutrophil-to-lymphocyte ratio (NLR), platelet-to-lymphocyte ratio (PLR) and lymphocyte-tomonocyte ratio (LMR), have been shown to be practical metrics for determining the prognosis in CRC. ${ }^{14-16}$

Tumour-associated inflammation and systemic inflammatory responses have emerged as critical components that govern the

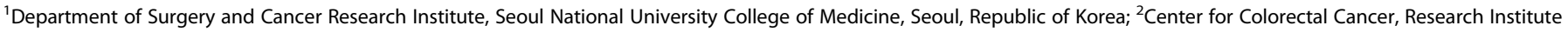
and Hospital, National Cancer Center, Goyang, Republic of Korea; ${ }^{3}$ Divison of Precision Medicine, Research Institute, National Cancer Center, Goyang, Republic of Korea;

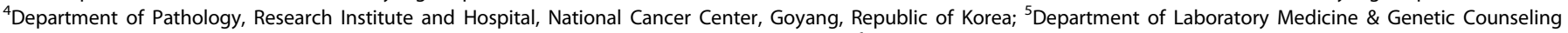

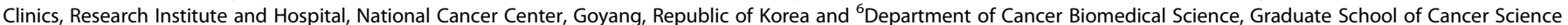
and Policy, National Cancer Center, Goyang, Republic of Korea

Correspondence: Hee Jin Chang (heejincmd@ncc.re.kr)

Received: 14 October 2019 Revised: 30 April 2020 Accepted: 13 May 2020

Published online: 3 June 2020 
clinical outcomes of CRC. However, little is known about the interrelationships and clinical impact of systemic cytokine profiles and inflammatory markers in CRC. This study aimed to investigate the association and prognostic impact of systemic cytokine profiles and inflammatory markers in CRC patients. In addition, the authors evaluated the prognostic value of the composite stratification of systemic cytokine profiles and inflammatory markers for predicting survival in CRC.

\section{METHODS}

Study population and blood collection

The study population consisted of patients with CRC who underwent surgery for primary CRC at the National Cancer Center, Korea. Participants in this study were prospectively recruited for blood sampling between March 2009 and September 2010. Patients who were older than 18 years were eligible. Patients with a previous or synchronous second primary malignancy and a previous history of colorectal surgery for CRC were excluded.

Approximately $5-10 \mathrm{ml}$ of blood was collected from eligible patients before surgery and after informed consent was obtained. Serum separator collection tubes were used for collecting blood, and were centrifuged at $2000 \mathrm{rpm}$ for $10 \mathrm{~min}$ at room temperature. The serum was extracted, dispensed into aliquots in polypropylene tubes and stored at $-70^{\circ} \mathrm{C}$. Each serum sample was assigned a unique identifier number to conceal the patient information.

Cytokine analysis and systemic inflammatory markers

Cytokines were assayed using a multiplex bead immunoassay system (Procarta Cytokine Assay Kit, Affymetrix eBioscience, Santa Clara, CA) according to the manufacturer's instructions in June 2012. Following incubation with multiple antibody-coated microbeads, multiple protein targets were quantified with a multi-analyte profiling technology by using a Luminex 100 (Luminex 100, Luminex Corporation, Austin, TX) and Bio-Plex software (Bio-Plex, Bio-Rad Laboratories Incorporated, Hercules, CA). The following seven cytokines, which are associated with immune cell-mediated inflammation and angiogenesis, were measured: proinflammatory cytokines (IL-1 $\beta$, IL- 6 and TNF- $\alpha$ ), inflammation-associated cytokines (IL-8 and IL-9), an antiinflammatory cytokine (IL-10) and an angiogenetic cytokine (VEGF-A). The assays were performed blinded to the clinical outcomes.

Clinicopathological data and preoperative laboratory results (including the neutrophil, lymphocyte, platelet and monocyte counts, fibrinogen, albumin and CRP levels) were collected. The modified Glasgow prognostic score (mGPS), NLR, PLR, LMR, prognostic nutritional index (PNI) and fibrinogen were assessed as systemic inflammatory markers. ${ }^{13-18}$ The mGPS was calculated based on the serum concentrations of CRP and albumin. ${ }^{13}$ Patients who had normal albumin $(>3.5 \mathrm{~g} / \mathrm{dl})$ and CRP $(<1.0 \mathrm{mg} / \mathrm{dl})$ levels were assigned a score of 0 . Patients with only elevated CRP (>1.0 mg/dl) were given a score of 1 . Those with low albumin $(<3.5 \mathrm{~g} / \mathrm{dl})$ and high CRP $(>1.0 \mathrm{mg} / \mathrm{dl})$ levels were given a score of 2. The NLR was defined as the absolute neutrophil count divided by the absolute lymphocyte count. The PLR was defined as the absolute platelet count divided by the absolute lymphocyte count. The LMR was defined as the absolute lymphocyte count divided by the absolute monocyte count. The PNI was determined using the following formula: albumin $(\mathrm{g} / \mathrm{l})+(5 \times$ total lymphocyte count $\left.\times 10^{3} / \mu \mathrm{l}\right){ }^{19}$

Immunohistochemistry for molecular subtype classification We classified 138 patients into consensus molecular subtypes (CMS) using immunohistochemistry according to Trinh's protocol. ${ }^{20}$ Tissue microarray blocks were previously constructed from 316 CRC cases resected in the National Cancer Center, Korea, from
2009 to 2010, and 138 out of 316 cases were matched with this study population. The blocks consisted of each representative tissue core ( $2 \mathrm{~mm}$ in diameter) taken from formalin-fixed paraffinembedded CRC tissues.

Immunohistochemical staining was performed using a BenchMark XT automated slide stainer (Ventana Medical Systems, Inc., Tucson, AZ, USA), by using anti-CDX2 (1:50, Cell Marque, EPR276RY), anti-ZEB1 (1:1000, Sigma, HPA027524) and antiHTR2B (1:500, Sigma, HPA012867) antibodies. Antigen retrieval

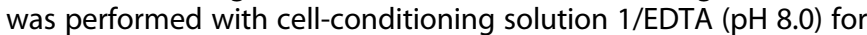
30 min at $98^{\circ} \mathrm{C}$ using the BenchMark staining module (Ventana Medical Systems, Inc.). Slides were counterstained with haematoxylin II and Bluing Reagent (cat. no. 760-2037, Ventana Medical Systems, Inc.) for $4 \mathrm{~min}$ at room temperature. For the negative control, tissue sections were incubated with Tris-buffered saline alone without the primary antibody.

Immunohistochemical expression was evaluated by two pathologists (HJC and NYH). CDX2 and HTR2B expression was quantitatively evaluated using a double-scoring system by estimating the staining intensity and percentage of stained cancer cells. The staining intensity was classified as 1 (weak), 2 (moderate) or 3 (strong). Immunoreactivity was scored as $0-300$ by multiplying the staining intensity by the percentage of cells stained. ZEB1 expression was evaluated by estimating the percentages of stromal nuclear expression within the entire tumour core, since the staining intensity of ZEB1 was relatively even (moderate) in the entire cases. Based on the mean values for expression of each marker, the staining results were classified into 'low' or 'high' expression. Epithelial-mesenchymal transition (EMT) phenotype was defined as 'low CDX2' and 'high ZEB1 and /or HTR2B'.

Microsatellite-instability phenotype was determined based on the results of immunohistochemistry for four mismatch repair proteins (MLH1, PMS2, MSH2 and MSH6). The antibodies were anti-MLH1 (Ready-to-use, Ventana, M1), anti-MSH2 (1:300, BD Pharmingen, G219-1129), anti-MSH6 (1:300, BD Pharmingen, 44/MSH6) and antiPMS2 (1:80, BD Pharmingen, A16-4). Loss of mismatch repair proteins was given if there was a distinct loss of nuclear staining in tumour cells, while normal stroma and lymphocytes showed strong nuclear staining in the same areas, thus excluding artefact and/or staining failure. Microsatellite instability was assigned in the case of the loss of any mismatch repair protein.

'Immune' (CMS1) subtype was a microsatellite-instable case. EMT phenotype was classified into 'mesenchymal' (CMS4) subtype, and the remaining cases were classified into 'epithelial' (CMS2/ CMS3) subtype.

\section{Immune score analysis}

To evaluate immune score in the tumour centre and invasive front, the whole tumour section was necessary, and 311 cases were available for the analysis. Immune score was evaluated by image analysis for CD3- and CD8-expressing lymphocytes in the tumour centre and invasive front, as described previously. ${ }^{21}$ Briefly, immunohistochemical stain was performed by using anti-CD3 (Ready-to-use, Ventana, 2GV6) and anti-CD8 (Ready-to-use, Ventana, SP57) antibodies, and the immunostained slides were scanned on an Aperio ScanScope CS instrument (Aperio Technologies, Inc.). CD3 + and CD8 + lymphocytes were automatically counted by the Nuclear $v 9$ algorithm of ImageScope ${ }^{T M}$ (Aperio Technologies, Inc.) image analysis system. We estimated four densities (number of positive cells per $\mathrm{mm}^{2}$ ) of $\mathrm{CD} 3+$ and CD8 + lymphocytes in the tumour centre and invasive front, respectively. If the density of both $\mathrm{CD}^{+}$and $\mathrm{CD}^{+} \mathrm{T}$ cells was elevated (higher than median) in both the centre and front, a high immune score was given.

DNA isolation

DNA was isolated from four $5-\mu \mathrm{m}$ slices of formalin-fixed, paraffinembedded (FFPE) tissues using the QIAamp DNA FFPE Tissue kit 
(Qiagen, Hilden, Germany) with the following amendments: samples were deparaffinised for $5 \mathrm{~min}$ with $20 \mathrm{~mL}$ of xylene (Merck KGaA, Darmstadt, Germany). Digestion steps were performed in double volumes, in that protease $\mathrm{K}$ digestion was performed in $200 \mu \mathrm{L}$ of ATL buffer, using $20 \mu \mathrm{L}$ of Protease $\mathrm{K}$ incubation at $65^{\circ} \mathrm{C}$ for $50 \mathrm{~min}$, followed by a heating step of $98^{\circ} \mathrm{C}$ for $1 \mathrm{~h}, 30 \mathrm{~min}$. RNA digestion was carried out, using $2 \mu \mathrm{L}$ of RNase A, then $200 \mu \mathrm{L}$ of $\mathrm{AL}$ buffer was added and $200 \mu \mathrm{L}$ of ethanol $(100 \%)$ was vortexed and loaded into columns in two steps. DNA was eluted in $20 \mu \mathrm{L}$ of nuclease-free water and quantified using a Nanodrop 2000 spectrophotometer (ThermoScientific, MA, USA).

Mutation analysis

To assess the relation between systemic markers and tumour molecular alteration, we performed somatic mutation analysis of seven genes associated with colorectal cancer (KRAS, BRAF, NRAS, APC, PIK3CA, PTEN and TP53).

Mutation detection was performed using iPLEX ${ }^{\circledast}$ Pro reagent on MassARRAY ${ }^{\oplus}$ System (Agena Bioscience, CA). The 53-hotspot target mutation sites of the following genes: APC, BRAF, KRAS, NRAS, PIK3CA, PTEN and TP53 for colon cancer were designed (Supplementary Table 1). About $10 \mathrm{ng}$ of genomic DNA was amplified using HotStarTaq polymerase (Qiagen, Valencia, CA), $100 \mathrm{~nm}$ of primers and $0.5 \mathrm{mM}$ dNTPs (Invitrogen, Inc.) on 9700 thermal cycler (Thermoscientific, MA, USA). PCR products were treated with SAP (Shrimp Alkaline Phosphatase) enzyme. Singlebase extension reaction was performed followed SAP treatment. The final product was cleaned with resin, and 16-nl product was transferred to a spectrochip using Nanodispenser RS 1000 (Agena Bioscience, CA). Finally, one nucleotide difference was detected using MALDI-TOF, and allele frequency was calculated using TYPER v4.0 software. This work was supported by the Genomics Core Facility in the National Cancer Center Korea.

\section{Follow-up}

After surgery, adjuvant chemotherapy was performed for patients with stage II with high risk, stage III and stage IV. The patients were followed up every 3 or 6 months for 5 years, and then every year thereafter. Physical examinations, chest X-ray, serum carcinoembryonic antigen (CEA) levels and abdominopelvic computed tomography were performed every 3 or 6 months. A colonoscopy was performed at 1 year after surgery, and every 2 years thereafter. Recurrence was detected by imaging tests, a biopsy or a combination of these methods.

Statistical analysis

The Reporting Recommendations for Tumor Marker Prognostic Studies (REMARK) criteria were taken into account in the study reporting (Supplementary Table 2). ${ }^{22}$ For comparison of the variables, Chi test, Mann-Whitney $U$ test or Kruskal-Wallis test were used according to the types of variables. Spearman's correlation tests were used to assess the pairwise relationship among cytokines and systemic inflammatory markers. The cut-off values of each cytokine and fibrinogen were determined by the median value. The cut-off values of systemic inflammatory markers were published in previous studies (NLR: 5, PLR: 150, LMR: 3 and PNI: 45). ${ }^{14,15,23,24}$ PFS was defined as the time to the recurrence or progression of CRC. OS was defined as the time to death of any cause. The log-rank test was used to compare the survival of patients with these variables. Using a forward selection method, Cox proportional hazard analysis was used for multivariable survival analysis, which included variables with values of $P<0.1$. Although no formal sample-size calculation was conducted in advance, the number of events (nearly 80 deaths and 120 progressions) compared with the number of Cox model variables (5 or 8 ) implied that the 'a minimum of 10 events per predictor' rule was exceeded, indicating the accuracy and precision of the regression estimates. ${ }^{25}$ All analyses were performed using SPSS version 22.0 (SPSS Inc., Chicago, IL, USA). A value of $P<0.05$ was considered statistically significant.

\section{RESULTS}

Among 870 patients who underwent surgery for CRC during the enrolment period, 168 patients with a previous or synchronous malignancy and 7 patients who were younger than 18 years were excluded. In the group of eligible patients, a total of 400 patients agreed to participate and were included in this study.

The clinicopathological characteristics of patients are shown in Table 1. The median age of the patients was 62 years [interquartile range (IQR): 53-69 years]. The median body mass index (BMI) was $23.6 \mathrm{~kg} / \mathrm{m}^{2}$ (IQR: $21.6-25.6 \mathrm{~kg} / \mathrm{m}^{2}$ ), and the median CEA level was $3.2 \mathrm{ng} / \mathrm{ml}$ (IQR: $2.0-7.0 \mathrm{ng} / \mathrm{ml}$ ).

The correlations within cytokines were moderate to strong for some cytokines (Fig. 1; Supplementary Table 3; IL-8 and IL-9: Spearman's correlation coefficient $[\rho]=0.71$, IL- 6 and IL-10: $\rho=$ 0.57 and IL- 6 and VEGF-A: $\rho=0.47$ ). There was also a moderate-tostrong correlation within some systemic inflammatory markers (NLR and LMR: $\rho=-0.66$, LMR and PNI: $\rho=0.55$ and NLR and PNI:

\begin{tabular}{|c|c|}
\hline Characteristics & $N=400$ \\
\hline \multicolumn{2}{|l|}{ Age } \\
\hline$\leq 60$ years & $186(46.5 \%)$ \\
\hline$>60$ years & $214(53.5 \%)$ \\
\hline \multicolumn{2}{|l|}{ Gender } \\
\hline Male & $244(61.0 \%)$ \\
\hline Female & $156(39.0 \%)$ \\
\hline \multicolumn{2}{|l|}{ Body mass index ${ }^{*}$} \\
\hline$\leq 25 \mathrm{~kg} / \mathrm{m}^{2}$ & 279 (69.9\%) \\
\hline$>25 \mathrm{~kg} / \mathrm{m}^{2}$ & $120(30.1 \%)$ \\
\hline \multicolumn{2}{|l|}{ Location } \\
\hline Colon & $211(52.8 \%)$ \\
\hline Rectum & 189 (47.3\%) \\
\hline \multicolumn{2}{|l|}{ Serum CEA } \\
\hline$\leq 5 \mathrm{ng} / \mathrm{mL}$ & $261(65.3 \%)$ \\
\hline$>5 \mathrm{ng} / \mathrm{mL}$ & 139 (34.8\%) \\
\hline \multicolumn{2}{|l|}{ Histologic grade ${ }^{\dagger}$} \\
\hline Low & $376(94.5 \%)$ \\
\hline High & $22(5.5 \%)$ \\
\hline \multicolumn{2}{|l|}{ Stage } \\
\hline 1 & $105(26.3 \%)$ \\
\hline 2 & $110(27.5 \%)$ \\
\hline 3 & $149(37.3 \%)$ \\
\hline 4 & $36(9.0 \%)$ \\
\hline \multicolumn{2}{|c|}{ Lymphovascular invasion" } \\
\hline No & $74(19.2 \%)$ \\
\hline Yes & $311(80.3 \%)$ \\
\hline \multicolumn{2}{|c|}{ Adjuvant chemotherapy } \\
\hline No & $135(33.8 \%)$ \\
\hline Yes & $265(66.3 \%)$ \\
\hline \multicolumn{2}{|c|}{ Neoadjuvant chemoradiotherapy } \\
\hline No & $361(90.2 \%)$ \\
\hline Yes & $39(9.8 \%)$ \\
\hline
\end{tabular}




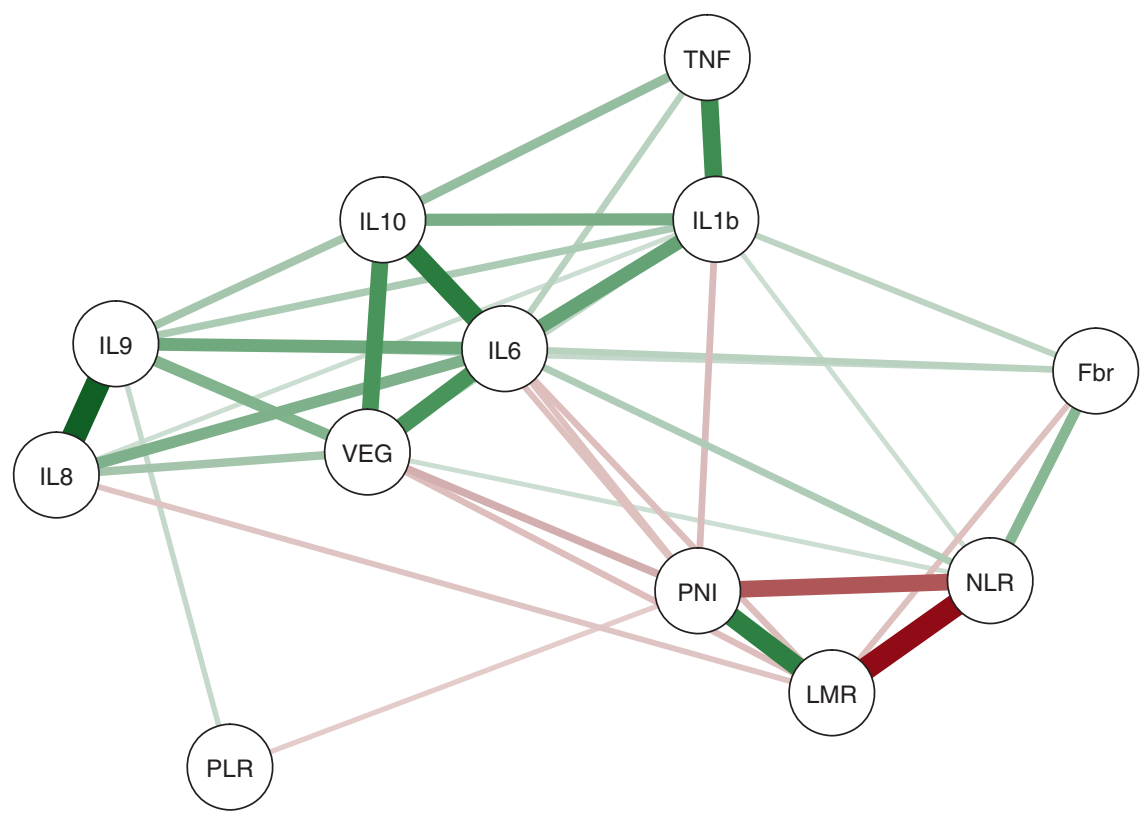

Fig. 1 Pairwise correlation graph between systemic cytokine profiles and inflammatory markers. Nodes represent systemic cytokine profiles or inflammatory markers, and lines represent their pairwise correlations. Only significant $(P<0.05)$ correlations are shown. Line thickness indicates the strength of Spearman's correlation. Green represents positive correlations, and red represents negative correlations. IL interleukin, TNF tumour necrosis factor, VEGF vascular endothelial growth factor, NLR neutrophil-to-lymphocyte ratio, PLR platelet-tolymphocyte ratio, LMR lymphocyte-to-monocyte ratio, PNI prognostic nutritional index, Fbr fibrinogen.

$\rho=-0.47)$. However, the associations between serum cytokines and systemic inflammatory markers were generally weak (Fig. 1, $-0.19 \leq \rho \leq 0.17)$.

The levels of cytokines and systemic inflammatory markers were not significantly different among immune (CMS1), epithelial (CMS2/ 3) and mesenchymal (CMS4) subtypes (Supplementary Table 4). KRAS mutation was related with low NLR, high LMR and high PNI (Supplementary Table 5). PIK3CA mutation was associated with high LNR and high PLR. TP53 mutation was related with low PLR, high LMR and high PNI. There were no significant systemic markers associated with BRAF, NRAS, APC and PTEN. Within tumourinfiltrating lymphocytes, there were strong correlations (Supplementary Fig. 1). There were only weak correlations between $\mathrm{CD}^{+} /$ $\mathrm{CD}^{+}{ }^{+}$T-cell counts and systemic inflammatory markers. However, there was no significant correlation between $\mathrm{CD}^{+} / \mathrm{CD}^{+}{ }^{+} \mathrm{T}$-cell counts and cytokines. High immune score was associated with low NLR, high PNI and low fibrinogen $(P=0.005, P=0.042$ and $P=$ 0.011 , respectively, Supplementary Table 6$)$. Microsatellite-instable tumour was associated with high NLR, high PLR and low PNI $(P=$ $0.033, P=0.041$ and $P=0.015$, respectively, Supplementary Table 6). However, cytokines were not related to tumour molecular alteration, immune score and microsatellite instability.

For survival analysis, patients were divided into low and high groups according to median values of cytokines and fibrinogen (Table 2). The median time of follow-up was 63.7 months (IQR: 49.0-84.0 months). In univariate survival analysis, IL-6, IL-8, VEGF$A$, the MGPS, NLR, LMR and fibrinogen were significantly associated with PFS and OS (Table 3). In multivariable analysis for PFS using variables with values of $P<0.1$, IL-8 (hazard ratio $[H R]=1.82,95 \%$ confidence interval $[\mathrm{Cl}]: 1.19-2.76)$, the LMR (HR $=0.52,95 \% \mathrm{Cl}: 0.35-0.79)$ and fibrinogen $(\mathrm{HR}=2.24,95 \%$ $\mathrm{Cl}$ : 1.41-3.55) were independent significant prognostic factors (Table 4). IL-8 (HR=2.33, 95\% Cl: 1.32-4.11) and the LMR (HR= $0.41,95 \% \mathrm{Cl}: 0.24-0.67)$ were independently and significantly associated with OS.

High concentration of IL-8 was associated with high CEA level. Patients with high LMR had higher body mass index and more colonic location than those with low LMR. Elevated fibrinogen

\begin{tabular}{|c|c|c|c|c|}
\hline & Median & IQR & Mean & SD \\
\hline $\mathrm{IL}-1 \beta, \mathrm{pg} / \mathrm{ml}$ & 2.47 & $1.94-3.28$ & 2.84 & 1.42 \\
\hline IL-6, pg/ml & 5.34 & $3.93-7.18$ & 5.86 & 3.04 \\
\hline IL-8, pg/ml & 4.17 & $2.81-8.72$ & 7.17 & 9.23 \\
\hline IL-9, pg/ml & 0.40 & $0.07-1.00$ & 0.87 & 1.55 \\
\hline IL-10, pg/ml & 1.69 & $1.46-2.01$ & 1.87 & 1.26 \\
\hline TNF- $\alpha, p g / m l$ & 8.15 & $5.87-11.8$ & 9.94 & 9.67 \\
\hline VEGF-A, pg/ml & 0.62 & $0.04-0.93$ & 0.78 & 0.97 \\
\hline NLR & 1.81 & $1.31-2.76$ & 2.29 & 1.66 \\
\hline PLR & 141.51 & $108.46-195.92$ & 161.69 & 79.33 \\
\hline LMR & 3.70 & $2.62-5.00$ & 3.97 & 1.87 \\
\hline $\mathrm{PNI}$ & 47.69 & $44.71-51.31$ & 48.10 & 5.02 \\
\hline Fibrinogen, mg/dl & 330.50 & $284.00-397.25$ & 350.38 & 95.02 \\
\hline
\end{tabular}

levels were correlated with older age, colonic location, high CEA, high stage and lymphovascular invasion (Supplementary Table 7).

To identify the effect of tumour molecular alterations on prognosis, survival analysis according to IL-8 and LMR was performed with stratification of molecular alterations. Because $B R A F, N R A S$ and PTEN had low rates of mutation, KRAS, APC, PIK3CA and TP53 were included for survival analysis. In patients without $A P C$ mutation, IL-8 and LMR were significant prognostic factors. However, those were not significant in patients with $A P C$ mutation (Supplementary Table 8). In terms of KRAS, PIK3CA and TP53 mutation status, IL-8 was a significant risk factor, regardless of mutation. On the other hand, LMR had inconsistent survival outcomes.

IL-8 and LMR were independent, significant factors for both PFS and OS. All patients were categorised into three groups based on 
Table 3. Univariate analysis for progression-free and overall survival.

\begin{tabular}{|c|c|c|c|c|c|}
\hline Variable & $N$ & 5YR-PFS & $P$ value & 5YR-OS & $P$ value \\
\hline Age & & & $<0.001$ & & $<0.001$ \\
\hline$\leq 60$ years & 186 & $78.9 \%$ & & $91.0 \%$ & \\
\hline$>60$ years & 214 & $65.3 \%$ & & $76.9 \%$ & \\
\hline Gender & & & 0.289 & & 0.310 \\
\hline Male & 244 & $70.6 \%$ & & $82.8 \%$ & \\
\hline Female & 156 & $73.5 \%$ & & $84.6 \%$ & \\
\hline Body mass index* & & & 0.685 & & 0.686 \\
\hline$\leq 25 \mathrm{~kg} / \mathrm{m}^{2}$ & 279 & $72.7 \%$ & & $83.1 \%$ & \\
\hline$>25 \mathrm{~kg} / \mathrm{m}^{2}$ & 120 & $69.4 \%$ & & $84.4 \%$ & \\
\hline Location & & & 0.055 & & 0.187 \\
\hline Colon & 211 & $78.0 \%$ & & $85.6 \%$ & \\
\hline Rectum & 189 & $67.3 \%$ & & $81.2 \%$ & \\
\hline Serum CEA & & & $<0.001$ & & 0.001 \\
\hline$\leq 5 \mathrm{ng} / \mathrm{mL}$ & 261 & $78.6 \%$ & & $88.5 \%$ & \\
\hline$>5 \mathrm{ng} / \mathrm{mL}$ & 139 & $58.9 \%$ & & $74.2 \%$ & \\
\hline Histologic grade ${ }^{\dagger}$ & & & 0.255 & & 0.003 \\
\hline Low & 376 & $72.5 \%$ & & $85.1 \%$ & \\
\hline High & 22 & $60.2 \%$ & & $59.8 \%$ & \\
\hline Stage & & & $<0.001$ & & $<0.001$ \\
\hline 1 & 105 & $89.8 \%$ & & $93.8 \%$ & \\
\hline 2 & 110 & $79.4 \%$ & & $91.9 \%$ & \\
\hline 3 & 149 & $67.1 \%$ & & $82.3 \%$ & \\
\hline 4 & 36 & $16.7 \%$ & & $31.1 \%$ & \\
\hline Lymphovascular invasion $^{\neq}$ & & & $<0.001$ & & 0.001 \\
\hline No & 74 & $88.7 \%$ & & $95.8 \%$ & \\
\hline Yes & 311 & $66.7 \%$ & & $80.1 \%$ & \\
\hline Adjuvant treatment & & & 0.128 & & 0.948 \\
\hline No & 135 & $76.9 \%$ & & $84.5 \%$ & \\
\hline Yes & 265 & $69.2 \%$ & & $83.0 \%$ & \\
\hline$I L-1 \beta$ & & & 0.909 & & 0.813 \\
\hline $\operatorname{Low}(\leq 2.47 \mathrm{pg} / \mathrm{ml})$ & 201 & $72.9 \%$ & & $85.4 \%$ & \\
\hline High (>2.47 pg/ml) & 199 & $70.5 \%$ & & $81.7 \%$ & \\
\hline IL-6 & & & $<0.001$ & & $<0.001$ \\
\hline Low $(\leq 5.34 \mathrm{pg} / \mathrm{ml})$ & 200 & $82.2 \%$ & & $90.9 \%$ & \\
\hline High (> 5.34 pg/ml) & 200 & $61.1 \%$ & & $75.9 \%$ & \\
\hline IL-8 & & & $<0.001$ & & $<0.001$ \\
\hline Low $(\leq 4.17 \mathrm{pg} / \mathrm{ml})$ & 201 & $81.7 \%$ & & $90.8 \%$ & \\
\hline High (>4.17 pg/ml) & 199 & $61.6 \%$ & & $76.2 \%$ & \\
\hline IL-9 & & & 0.151 & & 0.069 \\
\hline Low $(\leq 0.40 \mathrm{pg} / \mathrm{ml})$ & 199 & $73.8 \%$ & & $86.2 \%$ & \\
\hline High (>0.40 pg/ml) & 201 & $69.7 \%$ & & $80.7 \%$ & \\
\hline$I L-10$ & & & 0.682 & & 0.607 \\
\hline Low $(\leq 1.69 \mathrm{pg} / \mathrm{ml})$ & 209 & $69.7 \%$ & & $84.3 \%$ & \\
\hline High (>1.69 pg/ml) & 191 & $73.9 \%$ & & $82.7 \%$ & \\
\hline$T N F-a$ & & & 0.205 & & 0.431 \\
\hline Low $(\leq 8.15 \mathrm{pg} / \mathrm{ml})$ & 200 & $70.2 \%$ & & $83.8 \%$ & \\
\hline High (>8.15 pg/ml) & 200 & $73.2 \%$ & & $83.3 \%$ & \\
\hline$V E G F-A$ & & & 0.005 & & 0.045 \\
\hline Low $(\leq 0.62 \mathrm{pg} / \mathrm{ml})$ & 201 & $77.8 \%$ & & $87.2 \%$ & \\
\hline High (>0.62 pg/ml) & 199 & $65.5 \%$ & & $79.7 \%$ & \\
\hline$m G P S^{\S}$ & & & 0.012 & & 0.003 \\
\hline 0 & 292 & $75.6 \%$ & & $87.5 \%$ & \\
\hline 1 & 55 & $58.9 \%$ & & $72.9 \%$ & \\
\hline
\end{tabular}

\begin{tabular}{|c|c|c|c|c|c|}
\hline Variable & $N$ & 5YR-PFS & $P$ value & 5YR-OS & $P$ value \\
\hline 2 & 21 & $53.5 \%$ & & $62.6 \%$ & \\
\hline$N L R$ & & & 0.035 & & 0.011 \\
\hline$\leq 5$ & 380 & $73.0 \%$ & & $84.7 \%$ & \\
\hline$>5$ & 19 & $50.4 \%$ & & $57.4 \%$ & \\
\hline$P L R$ & & & 0.739 & & 0.523 \\
\hline$\leq 150$ & 218 & $72.7 \%$ & & $85.1 \%$ & \\
\hline$>150$ & 181 & $71.1 \%$ & & $81.7 \%$ & \\
\hline$L M R$ & & & 0.008 & & 0.003 \\
\hline$\leq 3$ & 140 & $64.6 \%$ & & $74.9 \%$ & \\
\hline$>3$ & 259 & $75.9 \%$ & & $88.1 \%$ & \\
\hline PNI & & & 0.816 & & 0.814 \\
\hline$\leq 45$ & 104 & $69.8 \%$ & & $81.8 \%$ & \\
\hline$>45$ & 295 & $72.6 \%$ & & $84.0 \%$ & \\
\hline Fibrinogen" & & & $<0.001$ & & $<0.001$ \\
\hline Low $(\leq 330.5 \mathrm{mg} / \mathrm{dl})$ & 193 & $83.7 \%$ & & $90.8 \%$ & \\
\hline High $(>330.5 \mathrm{mg} / \mathrm{dl})$ & 193 & $60.0 \%$ & & $75.7 \%$ & \\
\hline
\end{tabular}

their IL-8 and LMR levels: a low-risk group (low IL-8 and a high LMR), an intermediate-risk group (low IL-8 with a low LMR or high IL-8 with a high LMR) and a high-risk group (high IL-8 and a low LMR). Among these three groups, both PFS and OS were significantly different (Fig. $2, P<0.001$, respectively). The low-risk group had the best survival, and the high-risk group had the worst survival in terms of both PFS and OS (5-year PFS: $85.2 \%$ vs $57.4 \%$; 5-year PFS: $92.5 \%$ vs $66.1 \%$, respectively).

\section{DISCUSSION}

This study focused on the interrelationships and clinical impact of serum cytokine profiles and systemic inflammatory markers in CRC. It was demonstrated that serum cytokines and systemic inflammatory markers in CRC have moderate-to-strong intragroup correlations but only relatively weak intergroup correlations.

Both serum cytokines and systemic inflammatory markers were found to be associated with survival. The combination of these two factors can aid in the survival stratification of CRC patients.

Little research has been conducted exploring the relationship between serum cytokine profiles and systemic inflammatory markers in CRC. Chen et al. reported that a high NLR correlated with a distinct cytokine profile in metastatic CRC. ${ }^{26}$ In this study, the correlation between the NLR and cytokines was weak (IL-1 $\beta$ : $\rho=0.10$, IL-6: $\rho=0.17$ and VEGF-A: $\rho=0.10$ ). This difference may have been due to the different patients (metastatic vs all stages) and the type of variables (dichotomous vs continuous). Significant correlations among serum cytokine profiles and systemic inflammatory markers were evident, but the correlations between cytokines and systemic inflammatory markers were generally indistinct in this study. These results suggest that serum cytokine levels are not the only factors that influence systemic inflammatory markers. In Fig. 1, IL-6 had weak-to-strong correlations with all cytokines and systemic inflammatory markers, except the PLR. This body of work suggests that IL- 6 may be regarded as a central player in the systemic inflammation of CRC. 
Table 4. Multivariable analysis for progression-free and overall survival (forward selection).

\begin{tabular}{|c|c|c|c|c|c|c|}
\hline Variable & \multicolumn{3}{|c|}{ Progression-free survival* } & \multicolumn{3}{|c|}{ Overall survival $^{\dagger}$} \\
\hline Age & & & $<0.001$ & & & $<0.001$ \\
\hline$\leq 60$ years & Reference & & & Reference & & \\
\hline$>60$ years & 2.22 & $1.46-3.36$ & & 4.56 & $2.51-8.26$ & \\
\hline Location & & & 0.001 & & & \\
\hline Histologic grade & & & & & & 0.006 \\
\hline Low & & & & Reference & & \\
\hline High & & & & 2.90 & $1.35-6.25$ & \\
\hline Stage & & & $<0.001$ & & & $<0.001$ \\
\hline 1 & Reference & & & Reference & & \\
\hline Lymphovascular invasion & & & 0.043 & & & \\
\hline No & Reference & & & & & \\
\hline Yes & 2.48 & $1.03-5.96$ & & & & \\
\hline$I L-8$ & & & 0.005 & & & 0.003 \\
\hline Low $(\leq 4.17 \mathrm{pg} / \mathrm{ml})$ & Reference & & & Reference & & \\
\hline High (>4.17 pg/ml) & 1.82 & $1.19-2.76$ & & 2.33 & $1.32-4.11$ & \\
\hline$m G P S$ & & & 0.051 & & & \\
\hline 0 & Reference & & & & & \\
\hline 1 & 1.39 & $0.82-2.36$ & 0.224 & & & \\
\hline 2 & 0.47 & $0.20-1.07$ & 0.072 & & & \\
\hline$L M R$ & & & 0.002 & & & $<0.001$ \\
\hline
\end{tabular}

None of the cytokines and systemic inflammatory markers was related to pathologic molecular subtypes in this study. However, some of the systemic inflammatory markers were associated with tumour somatic mutation and tumour immune score unlike cytokines (Supplementary Tables 5 and 6). These results suggest that systemic cytokines were not directly related to the characteristics of primary tumour, but some of the systemic inflammatory markers were associated with primary tumour in terms of common molecular alteration and tumour response. The association between the molecular characteristics of primary tumour and cytokines or systemic inflammatory markers is not well known. Chen et al. showed that NLR was not related with tumour molecular alteration, including KRAS, NRAS, BRAF, PIK3CA mutation, PTEN loss and CIMP, in metastatic CRC. ${ }^{26}$ However, NLR was associated with KRAS and PIK3CA mutation in this study (Supplementary Table 5). These discrepancies may be from the dissimilarity in handling of LNR as a dichotomised or continuous variable because the absolute differences of NLR values between no mutation and mutation were relatively small in this study. Another study investigated the immunologic effect of SMAD4 on tumour microenvironment of $C R C .{ }^{27}$ SMAD4-negative $C R C$ had more tumour-infiltrating neutrophils with high expression of IL-8 than SMAD4-positive CRC. This result suggested that SMAD4negative CRC may be associated with high serum IL-8 level. Further researches using multi-omics data are required to reveal the relationship between markers of inflammation and tumour intrinsic characteristics.

It is not yet clear that systemic inflammation is related with local immunity. One study demonstrated that serum cytokines and tumour-infiltrating immune cells have high intragroup correlations but only relatively weak intergroup correlations. ${ }^{28}$ These results were consistent with those of the present study. Another study investigated the association between local immune cell density and systemic inflammatory makers. ${ }^{29}$ Among systemic inflammatory markers, LMR was significantly related with the CD3 + T cells in the core of the tumour. The other study demonstrated that high NLR was related with less lymphocytic reaction at the tumour-invasive margin. ${ }^{30}$ In the present study, high NLR and fibrinogen level were also associated with low immune score, while PNI was positively associated with immune score (Supplementary Table 6). These 


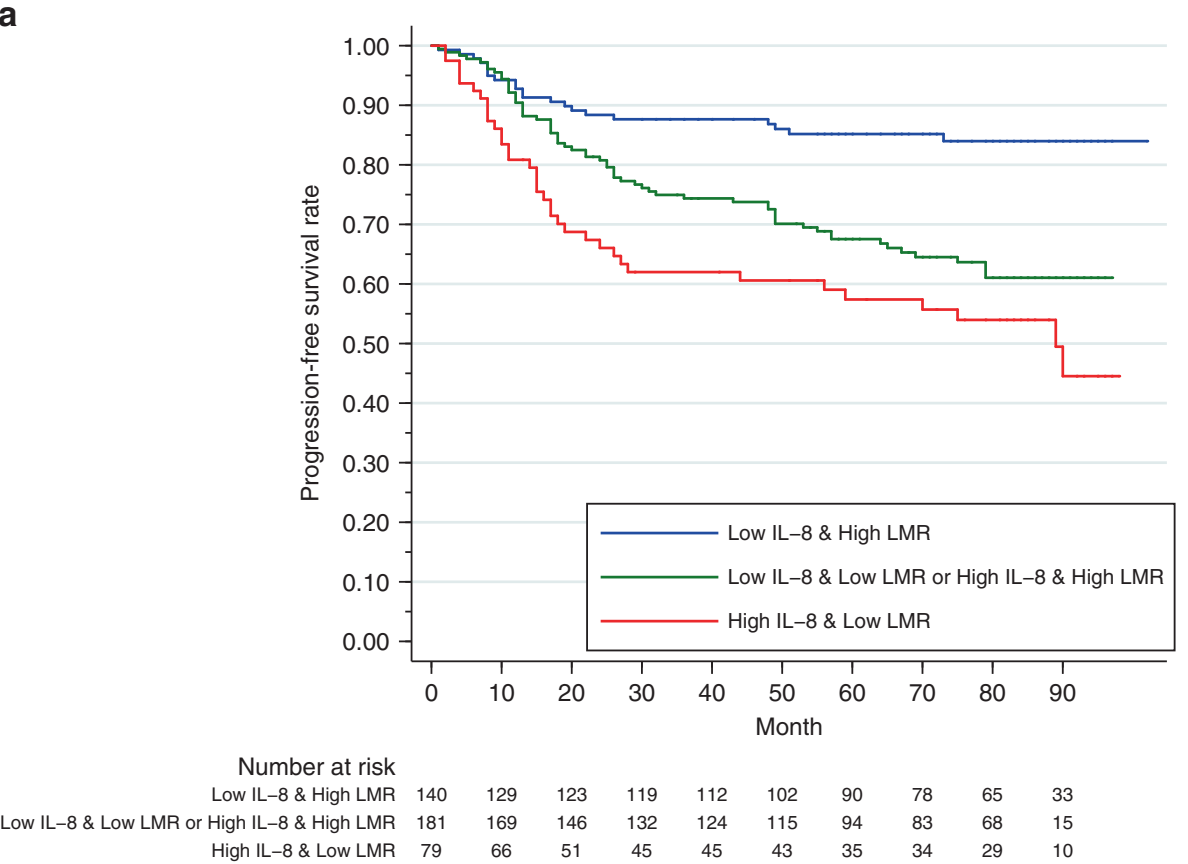

b

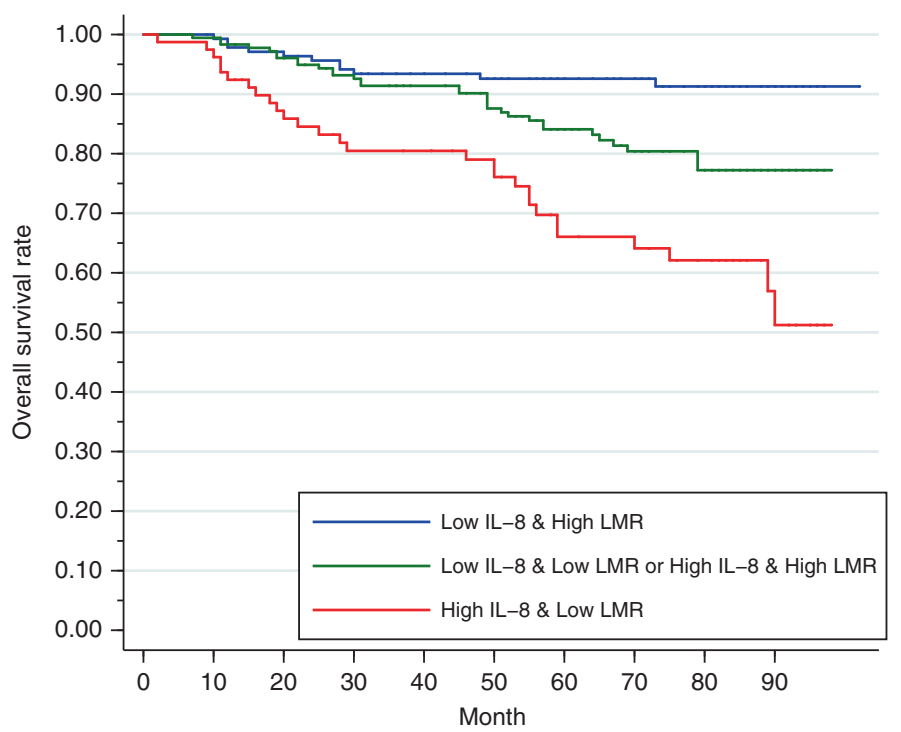

\begin{tabular}{|c|c|c|c|c|c|c|c|c|c|c|}
\hline Number at risk & & & & & & & & & & \\
\hline Low IL-8 \& High LMR & 140 & 138 & 133 & 127 & 118 & 108 & 90 & 78 & 65 & 33 \\
\hline Low IL-8 \& Low LMR or High IL-8 \& High LMR & 181 & 179 & 169 & 159 & 150 & 133 & 100 & 85 & 71 & 17 \\
\hline High IL-8 \& Low LMR & 79 & 77 & 66 & 58 & 57 & 54 & 35 & 34 & 29 & 10 \\
\hline
\end{tabular}

Fig. 2 Progression-free survival (PFS) and overall survival (OS) according to the combination of interleukin (IL)-8 and the lymphocyte-tomonocyte ratio (LMR). a PFS $(P<0.001$; low-risk group [low IL-8 and high LMR] vs intermediate-risk group [low IL-8 with low LMR or high IL-8 with high LMR], $P<0.001$; low- vs high-risk group [high IL-8 and low LMR], $P<0.001$; intermediate- vs high-risk group, $P=0.066)$. b OS $(P<$ 0.001 ; low- vs intermediate-risk group, $P=0.008$; low- vs high-risk group, $P<0.001$; intermediate- vs high-risk group, $P=0.002$ ).

results suggest that some of the systemic inflammatory markers could relate to local immunity in CRC. More studies using precise classification of immune cell categories may be needed (e.g. M1/M2 macrophage and N1/N2 neutrophil) to elucidate their relationship.

In the results of survival analysis-stratified tumour mutation, IL-8 and LMR were significant prognostic factors in patients without $A P C$ mutation, but not in patients with $A P C$ mutation. A study demonstrated that $A P C$ controls regulatory T-cell differentiation through microtubule-mediated nuclear factor of activated T-cell (NFAT) localisation in mouse model. ${ }^{31}$ APC mutation impairs T-cell activation and anti-inflammatory function. This impact of $A P C$ on the immune system and inflammation may affect the prognostic outcomes of IL-8 and LMR according to the status of APC mutation. Therefore, in specific tumour subtypes, cytokines and systemic inflammatory markers can be more effectively used as prognostic markers.

In the tumour microenvironment, IL- 6 acts as a pleiotropic proinflammatory cytokine, which has several main roles in cancer progression, migration and angiogenesis. ${ }^{32}$ Circulating IL-6 is associated with tumour stage, metastasis and survival. ${ }^{6}$ In a metaanalysis, patients with a high serum IL-6 level had a 1.76-fold higher risk of poor OS and a 2.97-fold higher risk of poor disease- 
free survival. ${ }^{33}$ In this study, IL-6 was associated with survival in univariate but not in multivariable analysis. Because there were significant correlations within cytokines and systemic inflammatory markers, a forward selection method was used in multivariable analysis to identify more robust prognostic factors. Although IL- 6 is a key mediator of tumour-associated inflammation, IL- 6 was not a stronger prognostic factor than IL-8 in multivariable analysis.

IL-8 is a proinflammatory CXC chemokine that is known to have tumorigenic and proangiogenic properties. ${ }^{34}$ The main functions of IL-8 are the promotion of neutrophil chemotaxis and angiogenic responses in endothelial cells. IL-8 is suggested to play a multifactorial role in the angiogenesis, tumour growth, metastasis and chemoresistance of tumours in vivo. ${ }^{35} \mathrm{IL}-8$ helps to recruit myeloid-derived suppressor cells, which inhibit T-cell proliferation and activation, into the tumour microenvironment in xenograft models. ${ }^{36}$ IL-8 was suggested as a potential prognostic marker in $\mathrm{CRC}^{8}$ In this study, high IL-8 was also independently associated with poor survival. IL-8 is correlated with tumour burden in xenograft models, and in patients with several cancer types. ${ }^{37} \mathrm{IL}-8$ could be an effective marker of early response to immunotherapy. ${ }^{38}$ Pharmaceutical agents inhibiting IL-8 may be a therapeutic option for targeting the tumour microenvironment. Ongoing clinical trials are evaluating the combination of anti-IL-8 antibody and immunotherapy for solid tumours. ${ }^{39,40}$

As a significant prognostic marker among systemic inflammatory markers in this study, the LMR consists of the lymphocyte count, which measures the degree of responsiveness of antitumour immune responses, and the monocyte count, which is an indicator of tumour proliferation. ${ }^{41}$ The monocyte count is supposed to reflect the formation of tumour-associated macrophages, which develop from circulating monocytes in the tumour microenvironment. ${ }^{42} \mathrm{~A}$ low LMR should have a low lymphocyte count or a high monocyte count. This low LMR could reflect an ineffective antitumour immune response and an elevated tumour burden. Thus, the LMR might be a strong predictor of prognosis in patients with CRC. A low LMR is associated with decreased survival in CRC. ${ }^{16}$

Fibrinogen was independently associated with survival in terms of only PFS. The role of fibrinogen as a promotor of tumour progression has been demonstrated in experimental studies, as it modulates angiogenesis and the metastasis of cancer cells. ${ }^{43}$ High-plasma fibrinogenaemia is significantly associated with worse survival in CRC. ${ }^{17,44}$

The mechanism explaining the association between systemic inflammation and survival outcome in CRC remains poorly understood. Tumour microenvironment may be one of the major factors in the mechanism. In tumour microenvironment, tumourassociated macrophages are derived and differentiated from circulating monocytes. Recent work has highlighted that tumourassociated macrophages enhance tumour progression and increase monocyte infiltration into the tumour site by $\mathrm{CCL} 8{ }^{45}$ Another study has reported that soluble factors released by CRC cells can change tumour-specific genetic signatures in circulating monocytes. ${ }^{46}$ Tumour necrosis can provoke systemic inflammatory response, and represent poor outcomes in CRC. ${ }^{47}$ Gurthrie and co-workers found that tumour necrosis is related with IL-6, IL10, VEGF and mGPS. $^{48}$ In these ways, systemic inflammation may be connected with tumour microenvironment.

In stage II CRC, to identify a high-risk group is clinically meaningful for the use of adjuvant treatment. IL-8, LMR and risk groups based on their IL-8 and LMR levels were investigated as prognostic factors in stage II patients. High IL-8 was associated with poor PFS $(P=0.040)$. Low LMR was related with poor OS $(P=$ $0.015)$. The low-risk group based on their IL-8 and LMR levels had better OS than the high-risk group $(P=0.025)$, and PFS than the intermediate-risk group $(P=0.048)$. Studies with larger sample sizes will be needed to examine these markers as prognostic factors in stage II CRC.
This study shows that the survival of CRC is not only determined by tumour characteristics, but also by inflammation and interactions between the tumour and the host. Systemic cytokine profiles and inflammatory markers, together with the current pathologic staging, provide complimentary predictive information for CRC.

To the best of our knowledge, this study was the first to investigate the relationship between serum cytokines and systemic inflammatory markers in CRC at all stages. The limitation of this study was that it assessed only seven cytokine markers. Future robust studies with many cytokine markers will reveal more information on the relationship between serum cytokines and systemic inflammatory markers. Another limitation was the inclusion of patients with neoadjuvant chemoradiotherapy. These patients may have different cytokine and systemic inflammatory profiles after neoadjuvant treatment. Although relatively small portions of patients (about 10\%) were treated with neoadjuvant treatment, this treatment can be a potential confounder.

In conclusion, the studied systemic cytokine profiles and inflammatory markers have relatively weak intergroup correlations. A composite classification of systemic cytokine profiles and inflammatory markers has increased prognostic value in $C R C$, specifically LMR and IL-8 values.

\section{ACKNOWLEDGEMENTS}

We thank all patients who contributed to this study.

\section{AUTHOR CONTRIBUTIONS}

J.W.P. and H.J.C. contributed to the study concept and design. H.Y.Y., N.Y.H. and H.J.C. contributed to data collection. J.W.P. analysed the data. J.W.P. and H.J.C. drafted the paper. B.C.K., S.Y.K., J.K. and J.H.O. provided clinical information, comments and improvements to the paper. All authors participated in the interpretation of the data and critically revised the paper.

\section{ADDITIONAL INFORMATION}

Ethics approval and consent to participate This study was approved by the Institutional Review Boards of National Cancer Center, Korea (IRB No. NCCCTS08351 and NCC2020-0051). All participants gave their signed informed consent, and all samples were obtained with the patients' informed consent. The study was performed in accordance with the Declaration of Helsinki.

Consent to publish Not applicable.

Data availability The data sets used and analysed during this study are available from the corresponding author on reasonable request.

Competing interests The authors declare no competing interests.

Funding information This study was supported by a grant from the National Cancer Centre of Korea (NCC-1910213 and NCC-2010371).

Supplementary information is available for this paper at https://doi.org/10.1038/ s41416-020-0924-5.

Note This work is published under the standard license to publish agreement. After 12 months the work will become freely available and the license terms will switch to a Creative Commons Attribution 4.0 International (CC BY 4.0).

Publisher's note Springer Nature remains neutral with regard to jurisdictional claims in published maps and institutional affiliations.

\section{REFERENCES}

1. Diakos, C. I., Charles, K. A., McMillan, D. C. \& Clarke, S. J. Cancer-related inflammation and treatment effectiveness. Lancet Oncol. 15, e493-e503 (2014).

2. Pages, F., Mlecnik, B., Marliot, F., Bindea, G., Ou, F. S., Bifulco, C. et al. International validation of the consensus immunoscore for the classification of colon cancer: a prognostic and accuracy study. Lancet 391, 2128-2139 (2018). 
3. Gunawardene, A., Dennett, E. \& Larsen, P. Prognostic value of multiple cytokine analysis in colorectal cancer: a systematic review. J. Gastrointest. Oncol. 10, 134-143 (2019)

4. Sylman, J. L., Mitrugno, A., Atallah, M., Tormoen, G. W., Shatzel, J. J., Tassi Yunga, S. et al. The predictive value of inflammation-related peripheral blood measurements in cancer staging and prognosis. Front. Oncol. 8, 78 (2018).

5. West, N. R., McCuaig, S., Franchini, F. \& Powrie, F. Emerging cytokine networks in colorectal cancer. Nat. Rev. Immunol. 15, 615-629 (2015).

6. Knupfer, H. \& Preiss, R. Serum interleukin- 6 levels in colorectal cancer patients-a summary of published results. Int. J. Colorectal Dis. 25, 135-140 (2010).

7. Itoh, Y., Joh, T., Tanida, S., Sasaki, M., Kataoka, H., Itoh, K. et al. IL-8 promotes cell proliferation and migration through metalloproteinase-cleavage proHB-EGF in human colon carcinoma cells. Cytokine 29, 275-282 (2005).

8. Xia, W., Chen, W., Zhang, Z., Wu, D., Wu, P., Chen, Z. et al. Prognostic value, clinicopathologic features and diagnostic accuracy of interleukin-8 in colorectal cancer: a meta-analysis. PLOS ONE 10, e0123484 (2015).

9. Mager, L. F., Wasmer, M. H., Rau, T. T. \& Krebs, P. Cytokine-induced modulation of colorectal cancer. Front. Oncol. 6, 96 (2016).

10. Kwon, K. A., Kim, S. H., Oh, S. Y., Lee, S., Han, J. Y., Kim, K. H. et al. Clinical significance of preoperative serum vascular endothelial growth factor, interleukin-6, and C-reactive protein level in colorectal cancer. BMC Cancer 10, 203 (2010).

11. Werther, K., Christensen, I. J. \& Nielsen, H. J. Danish RANX05 Colorectal Cancer Study Group. Prognostic impact of matched preoperative plasma and serum VEGF in patients with primary colorectal carcinoma. Br. J. Cancer 86, 417-423 (2002).

12. Akgul, O., Cetinkaya, E., Yalaza, M., Ozden, S. \& Tez, M. Prognostic efficacy of inflammation-based markers in patients with curative colorectal cancer resection. World J. Gastrointest. Oncol. 9, 300-307 (2017).

13. Proctor, M. J., Morrison, D. S., Talwar, D., Balmer, S. M., O'Reilly, D. S., Foulis, A. K. et al. An inflammation-based prognostic score (mGPS) predicts cancer survival independent of tumour site: a Glasgow Inflammation Outcome Study. Br. J. Cancer 104, 726-734 (2011).

14. Li, M. X., Liu, X. M., Zhang, X. F., Zhang, J. F., Wang, W. L., Zhu, Y. et al. Prognostic role of neutrophil-to-lymphocyte ratio in colorectal cancer: a systematic review and meta-analysis. Int. J. Cancer 134, 2403-2413 (2014).

15. Lu, C., Gao, P., Yang, Y., Chen, X., Wang, L., Yu, D. et al. Prognostic evaluation of platelet to lymphocyte ratio in patients with colorectal cancer. Oncotarget 8 , 86287-86295 (2017)

16. Song, W., Wang, K., Zhang, R. J. \& Zou, S. B. Prognostic value of the lymphocyte monocyte ratio in patients with colorectal cancer: a meta-analysis. Medicine $\mathbf{9 5}$, e5540 (2016).

17. Son, H. J., Park, J. W., Chang, H. J., Kim, D. Y., Kim, B. C., Kim, S. Y. et al. Preoperative plasma hyperfibrinogenemia is predictive of poor prognosis in patients with nonmetastatic colon cancer. Ann. Surg. Oncol. 20, 2908-2913 (2013).

18. Sun, G., Li, Y., Peng, Y., Lu, D., Zhang, F., Cui, X. et al. Impact of the preoperative prognostic nutritional index on postoperative and survival outcomes in colorectal cancer patients who underwent primary tumor resection: a systematic review and meta-analysis. Int. J. Colorectal Dis. 34, 681-689 (2019).

19. Sagawa, M., Katsube, T., Konno, S., Murayama, M., Yamaguchi, K., Isohata, N. et al. The significance of Onodera's prognostic nutritional index for the treatment of gastrointestinal cancer. Gan Kagaku Ryoho 35, 2253-2255 (2008).

20. Trinh, A., Trumpi, K., De Sousa, E. M. F., Wang, X., de Jong, J. H., Fessler, E. et al. Practical and robust identification of molecular subtypes in colorectal cancer by immunohistochemistry. Clin. Cancer Res. 23, 387-398 (2017).

21. Kwak, Y., Koh, J., Kim, D. W., Kang, S. B., Kim, W. H. \& Lee, H. S. Immunoscore encompassing $\mathrm{CD} 3+$ and $\mathrm{CD} 8+\mathrm{T}$ cell densities in distant metastasis is a robust prognostic marker for advanced colorectal cancer. Oncotarget 7, 81778-81790 (2016).

22. Altman, D. G., McShane, L. M., Sauerbrei, W. \& Taube, S. E. Reporting recommendations for tumor marker prognostic studies (REMARK): explanation and elaboration. PLoS Med. 9, e1001216 (2012).

23. Wu, Q., Hu, T., Zheng, E., Deng, X. \& Wang, Z. Prognostic role of the lymphocyteto-monocyte ratio in colorectal cancer: an up-to-date meta-analysis. Medicine $\mathbf{9 6}$, e7051 (2017).

24. Yang, Y., Gao, P., Chen, X., Song, Y., Shi, J., Zhao, J. et al. Prognostic significance of preoperative prognostic nutritional index in colorectal cancer: results from a retrospective cohort study and a meta-analysis. Oncotarget 7, 58543-58552 (2016).

25. Peduzzi, P., Concato, J., Feinstein, A. R. \& Holford, T. R. Importance of events per independent variable in proportional hazards regression analysis. II. Accuracy and precision of regression estimates. J. Clin. Epidemiol. 48, 1503-1510 (1995).

26. Chen, Z. Y., Raghav, K., Lieu, C. H., Jiang, Z. Q., Eng, C., Vauthey, J. N. et al. Cytokine profile and prognostic significance of high neutrophil-lymphocyte ratio in colorectal cancer. Br. J. Cancer 112, 1088-1097 (2015).
27. Ogawa, R., Yamamoto, T., Hirai, H., Hanada, K., Kiyasu, Y., Nishikawa, G. et al. Loss of SMAD4 promotes colorectal cancer progression by recruiting tumor-associated neutrophils via the CXCL1/8-CXCR2 axis. Clin. Cancer Res. 25, 2887-2899 (2019).

28. Väyrynen, J. P., Kantola, T., Väyrynen, S. A., Klintrup, K., Bloigu, R., Karhu, T. et al. The relationships between serum cytokine levels and tumor infiltrating immune cells and their clinical significance in colorectal cancer. Int. J. Cancer 139, 112-121 (2016).

29. Guo, G., Wang, Y., Zhou, Y., Quan, Q., Zhang, Y., Wang, H. et al. Immune cell concentrations among the primary tumor microenvironment in colorectal cancer patients predicted by clinicopathologic characteristics and blood indexes. J. Immunother. Cancer 7, 179 (2019).

30. Pine, J. K., Morris, E., Hutchins, G. G., West, N. P., Jayne, D. G., Quirke, P. et al. Systemic neutrophil-to-lymphocyte ratio in colorectal cancer: the relationship to patient survival, tumour biology and local lymphocytic response to tumour. Br. J. Cancer 113, 204-211 (2015).

31. Agüera-González, S., Burton, O. T., Vázquez-Chávez, E., Cuche, C., Herit, F., Bouchet, J. et al. Adenomatous polyposis coli defines treg differentiation and antiinflammatory function through microtubule-mediated NFAT localization. Cell Rep. 21, 181-194 (2017).

32. Taniguchi, K. \& Karin, M. IL-6 and related cytokines as the critical lynchpins between inflammation and cancer. Semin. Immunol. 26, 54-74 (2014).

33. Xu, J., Ye, Y., Zhang, H., Szmitkowski, M., Makinen, M. J., Li, P. et al. Diagnostic and prognostic value of serum interleukin-6 in colorectal cancer. Medicine 95, e2502 (2016).

34. Waugh, D. J. \& Wilson, C. The interleukin-8 pathway in cancer. Clin. Cancer Res. 14, 6735-6741 (2008).

35. Ning, Y., Manegold, P. C., Hong, Y. K., Zhang, W., Pohl, A., Lurje, G. et al. Interleukin-8 is associated with proliferation, migration, angiogenesis and chemosensitivity in vitro and in vivo in colon cancer cell line models. Int. J. Cancer 128, 2038-2049 (2011).

36. Alfaro, C., Teijeira, A., Onate, C., Perez, G., Sanmamed, M. F., Andueza, M. P. et al. Tumor-produced interleukin-8 attracts human myeloid-derived suppressor cells and elicits extrusion of neutrophil extracellular traps (NETs). Clin. Cancer Res. 22, 3924-3936 (2016)

37. Sanmamed, M. F., Carranza-Rua, O., Alfaro, C., Onate, C., Martin-Algarra, S., Perez, G. et al. Serum interleukin-8 reflects tumor burden and treatment response across malignancies of multiple tissue origins. Clin. Cancer Res. 20, 5697-5707 (2014).

38. Alfaro, C., Sanmamed, M. F., Rodriguez-Ruiz, M. E., Teijeira, A., Onate, C., Gonzalez, A. et al. Interleukin-8 in cancer pathogenesis, treatment and follow-up. Cancer Treat. Rev. 60, 24-31 (2017).

39. Charles, G. D., Bristol-Myers, S. \& Columbia, U. Nivolumab and BMS- 986253 for hormone-sensitive prostate cancer (MAGIC-8). https://ClinicalTrials.gov/show/ NCT03689699 (2020).

40. Thomas, M. \& Bristol-Myers, S. Icahn School of Medicine at Mount S, Bristol-Myers S. Neoadjuvant nivolumab with CCR2/5-inhibitor or Anti-IL-8) for non-small cell lung cancer (NSCLC) or hepatocellular carcinoma (HCC). https://ClinicalTrials.gov/ show/NCT04123379 (2020).

41. Hu, S., Zou, Z., Li, H., Zou, G., Li, Z., Xu, J. et al. The preoperative peripheral blood monocyte count is associated with liver metastasis and overall survival in colorectal cancer patients. PLOS ONE 11, e0157486 (2016).

42. Condeelis, J. \& Pollard, J. W. Macrophages: obligate partners for tumor cell migration, invasion, and metastasis. Cell 124, 263-266 (2006).

43. Staton, C. A., Brown, N. J. \& Lewis, C. E. The role of fibrinogen and related fragments in tumour angiogenesis and metastasis. Expert. Opin. Biol. Ther. 3, 1105-1120 (2003).

44. Li, M., Wu, Y., Zhang, J., Huang, L., Wu, X. \& Yuan, Y. Prognostic value of pretreatment plasma fibrinogen in patients with colorectal cancer: a systematic review and meta-analysis. Medicine 98, e16974 (2019).

45. Cassetta, L., Fragkogianni, S., Sims, A. H., Swierczak, A., Forrester, L. M., Zhang, H. et al. Human tumor-associated macrophage and monocyte transcriptional landscapes reveal cancer-specific reprogramming, biomarkers, and therapeutic targets. Cancer Cell 35, 588-602 (2019).

46. Hamm, A., Prenen, H., Van Delm, W., Di Matteo, M., Wenes, M., Delamarre, E. et al. Tumour-educated circulating monocytes are powerful candidate biomarkers for diagnosis and disease follow-up of colorectal cancer. Gut 65, 990-1000 (2016).

47. Richards, C. H., Roxburgh, C. S., Anderson, J. H., McKee, R. F., Foulis, A. K., Horgan, P. G. et al. Prognostic value of tumour necrosis and host inflammatory responses in colorectal cancer. Br. J. Surg. 99, 287-294 (2012).

48. Guthrie, G. J., Roxburgh, C. S., Richards, C. H., Horgan, P. G. \& McMillan, D. C. Circulating IL-6 concentrations link tumour necrosis and systemic and local inflammatory responses in patients undergoing resection for colorectal cancer. Br. J. Cancer 109, 131-137 (2013). 